\title{
EFECTO DE LA COBERTURA FORESTAL SOBRE LAS CARACTERÍSTICAS DEL SUELO EN LA FINCA EXPERIMENTAL INTERDISCIPLINARIA DE MODELOS AGROECOLÓGICOS: UN ESTUDIO DE CASO
}

\author{
EFFECT OF FOREST COVER ON SOIL CHARACTERISTICS AT THE \\ FINCA EXPERIMENTAL INTERDISCIPLINARIA DE MODELOS \\ AGROECOLÓGICOS: A CASE STUDY
}

Josué Mora-Chacón ${ }^{1}$

Shiori Nakajima ${ }^{2}$

Pedro A. Rojas ${ }^{3}$

Carlos Rojas ${ }^{4}$

Recibido: 10.05 .20

Aprobado: 23.11.19

DOI: $10.15517 /$ isucr.v20i42.41851

\section{Resumen}

La gestión del suelo es primaria en el contexto de adaptación al cambio climático. Sin embargo, para poder determinar su manejo, uso y conservación, se requiere de información primaria que ofrezca los insumos necesarios para la toma de decisiones. Este proyecto respondió a la poca documentación base en la sección forestal de la Finca Experimental Interdisciplinaria de Modelos

\footnotetext{
${ }^{1}$ Costarricense, ingeniero agrícola, estudiante de Escuela de Ingeniería de Biosistemas, Universidad de Costa Rica, email: josue.morachacon@ucr.ac.cr

2 Japonesa, ingeniera forestal, voluntaria de Agencia del Gobierno Japonés para la Cooperación Internacional (JICA), email: shiori.nakajima@ucr.ac.cr

${ }^{3}$ Costarricense, técnico de laboratorio, Instituto de Investigaciones en Ingeniería, Universidad de Costa Rica, email: pedro.rojas@ucr.ac.cr

${ }^{4}$ Costarricense, investigador, Instituto de Investigaciones en Ingeniería/Finca Experimental Interdisciplinaria de Modelos Agroecológicos, Universidad de Costa Rica, email: carlos.rojasalvarado@ucr.ac.cr
} 
Agroecológicos en Turrialba, Costa Rica. En este estudio se analizó el efecto del uso del suelo sobre características fisicoquímicas de este último para contrastar los procesos asociados con la gestión forestal y comprender el efecto temporal de la zonificación sobre las variables estudiadas. Una serie de variables biológicas también fueron determinadas para evaluar uniformidad de respuestas dentro del bosque. La textura del suelo en toda el área de estudio se clasificó en clase media a gruesa y la fertilidad química fue determinada en media a alta sin mayores diferencias entre secciones de la finca. Sin embargo, la coloración, la densidad aparente $\left(0.6-1.4 \mathrm{~g} / \mathrm{cm}^{3}\right)$, la porosidad (48.1-77.6\%) y la velocidad de infiltración $(552-3405 \mathrm{~mm} /$ día) fueron influenciadas por la textura y el uso del suelo. El promedio de respiración del suelo (46.33 $\left.\mu \mathrm{g} \mathrm{C}-\mathrm{CO}_{2} / \mathrm{gh}\right)$ fue alto y no se observaron diferencias en las variables biológicas dentro del bosque. La información obtenida en este estudio demostró que la cobertura forestal ha modificado en mayor grado las variables estructurales con respecto a las otras categorías de uso del suelo acá estudiadas. El bosque, sin embargo, pareciera haberse desarrollado como una unidad funcional homogénea.

Palabras clave: cambio climático; gestión ambiental; ingeniería ambiental; seguimiento; SIG; uso de la tierra.

\begin{abstract}
Soil management is essential in the context of climate change adaptation, but baseline information is first required for its management, use and conservation. This project aimed to address the poor documentation of the soil in the forest section of the Finca Experimental Interdisciplinaria de Modelos Agroecológicos in Turrialba, Costa Rica. The aim of this study was to analyze the effect of land use on physicochemical characteristics of the soil to contrast the processes associated with forest management and evaluate the temporal effect of zonification on soil characteristics. A series of biological variables were also determined to evaluate response uniformity within the forest. For all the studied area, soil texture was classified as medium and coarse and chemical fertility was medium to high without major differences among land use sections. However, color, apparent density $\left(0.6-1.4 \mathrm{~g} / \mathrm{cm}^{3}\right)$, porosity $(48.2-77.7 \%)$ and infiltration velocity $(552-3405 \mathrm{~mm} /$ day $)$ were influenced by soil texture and land use. Soil respiration had a high average of $46.33 \mu \mathrm{g} \mathrm{C}-\mathrm{CO}_{2} / \mathrm{gh}$ and no differences in biological variables were observed within the forest. The information obtained in this study demonstrated that forest cover has had a stronger impact on structural variables in relation with other land use types. The forest, however, seemed to have developed as a functional homogeneous unit.
\end{abstract}

Keywords: climate change; environmental engineering; environmental management; GIS; land use; monitoring.

El suelo constituye uno de los recursos más importantes para la subsistencia humana en el planeta ya que representa la base fundamental para la producción agrícola, forestal y pecuaria (Wall, Ritz, Six, Strong, y van der Putten, 2012). Dentro del contexto de la adaptación al cambio climático, el estudio comparativo de suelos para facilitar estrategias de uso sostenible y conservación funcional es estratégico (ver Codur \& Watson, 2018). Debido a que el suelo se puede 
considerar como una matriz de factores multidimensionales con heterogeneidad de patrones (Wieder et al., 2015), es importante a su vez documentar los procesos de transformación en este recurso para afinar la futura planificación de su uso.

Cuando existe una cobertura forestal sobre el suelo, la vegetación juega un papel importante en el establecimiento y transformación de este (Stocking, 1988). La materia vegetal en descomposición altera la estructura y función de los biosistemas presentes y como consecuencia se da una perturbación del horizonte superficial (Bautista-Cruz, Gutiérrez-Castorena, CastilloSánchez, y Etchevers-Barra, 2005). Al mismo tiempo, la drástica disminución en la radiación incidente con un efecto primario en la temperatura y la humedad del suelo, permiten una mayor complejidad en las redes tróficas presentes (Barnes et al., 2017). Este potencial incremento en la riqueza bioquímica favorece que tal recurso, en condiciones forestales, se desarrolle de forma diferente con relación a otros tipos de uso del suelo (Salmon, 2018).

Este proyecto se ha diseñado como parte de las gestiones para documentar la Finca Experimental Interdisciplinaria de Modelos Agroecológicos. Esta estación experimental, de reciente consolidación (Resolución R-27-2014, 2014), es administrada por la Universidad de Costa Rica y se encuentra en los terrenos del botadero de material extraído durante la construcción de túneles del proyecto hidroeléctrico Angostura a principios de los años 90 (Consejo Universitario, 2009, p. 46). Por su origen, el suelo de esta finca corresponde con las capas profundas originalmente consolidadas en las márgenes del Río Reventazón. Con base en la sectorización original que el Instituto Costarricense de Electricidad implementó en estas tierras, además de zonas agrícolas y silvopastoriles, alrededor del $60 \%$ de la finca fue reforestado y separado para protección forestal (Consejo Universitario, 2009, p. 46-47). Como consecuencia, los cambios en el uso de la tierra en los últimos 30 años han sido mínimos y la sectorización original todavía se mantiene.

De esta forma, la estación experimental en cuestión representa un laboratorio natural para el estudio de fenómenos de gestión del uso de la tierra, dentro de los cuales, el suelo es un componente necesario de documentar (ver Lal, 2016). Por tanto, el objetivo principal del presente proyecto ha sido caracterizar en su nivel primario, de forma fisicoquímica, el suelo de toda la estación experimental y contrastar la información obtenida entre los diferentes tipos de uso del suelo haciendo énfasis en la zona forestal. De esta forma, se ha pretendido evaluar el efecto de la cobertura forestal y gestión diferenciada dada por la zonificación existente, a lo largo de un poco 
más de un cuarto de siglo, sobre las variables estudiadas. Debido a que tal caracterización primaria del suelo para toda la finca correspondía con un objetivo no cumplido hasta el momento, el presente proyecto también se ha visualizado como un esfuerzo hacia la adaptación al cambio climático y a la consolidación de estrategias agrícolas integradas con servicios ecosistémicos. Estas últimas dos ideas, corresponden con los objetivos de creación de la estación experimental en estudio (Consejo Universitario, 2009, p. 47).

Materiales y métodos

Este proyecto se llevó a cabo entre los años 2018 y 2019 en la Finca Experimental Interdisciplinaria de Modelos Agroecológicos (FEIMA), en Turrialba, Costa Rica. Este sitio está rodeado de tierras agrícolas y según la demarcación original está dividido en secciones de a) agricultura, b) ganadería y c) bosque. La última sección corresponde a un parche forestal en regeneración dominado por árboles de Erythrina peoppigiana (Walp.) O.F. Cook.

Por medio de fotografías satelitales se realizó una división espacial de la finca en parcelas de alrededor de 100x100 m. Con esta estrategia a priori se seleccionó un punto de muestreo por parcela que, en algunos casos, por efecto del borde de la propiedad, no quedó localizado en el centro de esta. Tal proceso aleatorio, generado en el software QGIS permitió minimizar el sesgo de selección de los 45 puntos de estudio y definió 28 parcelas de bosque, 10 de agricultura y 7 de ganadería. La sección de bosque fue subdividida tres zonas denominadas a) sureste, b) media y c) noroeste para efectos de análisis.

En cada punto de análisis se tomaron dos muestras de suelo a $15 \mathrm{~cm}$ de profundidad con un barreno sencillo. Para ello se utilizó la técnica compuesta después de haber limpiado el área de cobertura vegetal. Una serie de muestras paralelas a las anteriores fueron recolectadas de forma similar, a $20 \mathrm{~cm}$ de profundidad, con un barreno de doble anillo. Con las primeras se determinó la textura, el color y las propiedades químicas del suelo. Con las segundas se determinó la densidad aparente y la porosidad. Además, en ocho puntos determinados al azar, con representación de todas las secciones, se caracterizaron la velocidad de infiltración y la infiltración acumulada usando el método de Porchet (ver Kessler \& Oosterbaan, 1974).

La textura fue establecida, tras haber secado las muestras por $24 \mathrm{~h}$ y eliminar granulometrías mayores a 2 mm, con el método de medición en recipiente (ver Whiting, Wilson, y Card, 2003). El 
color fue determinado en estado húmedo y en estado físico (seco) según la notación Munsell. Las propiedades químicas, asociadas con los valores de pH, CICE, saturación de acidez, materia orgánica, C, N, Ca, Mg, K, P, Zn, Fe, Cu y Mn, fueron determinadas por el Laboratorio de Suelos del Centro de Investigaciones Agronómicas (CIA) de la Universidad de Costa Rica por medio de la solución extractora $\mathrm{KCl}$ - Olsen Modificado. Con la textura, se calculó la capacidad de campo y el punto de marchitez permanente utilizando la metodología de Saxton, Rawls, Romberger, y Papendick (1986). Con las propiedades químicas se determinó la fertilidad a partir de los estándares para plantaciones de café (Méndez \& Bertsch, 2012). La densidad aparente fue determinada según el método de Forsythe (1985) y la porosidad fue calculada a partir de la anterior, utilizando un valor de $2.65 \mathrm{~g} / \mathrm{cm}^{3}$ como valor de densidad real.

Para evaluar uniformidad de respuesta dentro del parche boscoso se hizo además una determinación de la respiración del suelo y se calculó la diversidad de plántulas y la de mixomicetes. Este último grupo de microorganismos corresponde con amebas de vida libre presentes en suelos y es útil para establecer salud del suelo (ver Stephenson, Fiore-Donno, y Schnittler, 2011; Sibaja-Matarrita, Barboza-Chinchilla, y Rojas, 2018;). Lo anterior se llevó a cabo en una selección de dos puntos correspondientes con cada una de las tres zonas de bosque, para un total de seis puntos de muestreo. La respiración de suelo se registró por triplicado con una variación del método de Moebius-Clune et al. (2016) en la que la determinación de $\mathrm{CO}_{2}$ se hizo por valoración de $\mathrm{NaOH}$ con $\mathrm{HCl}$ de concentración conocida a partir de la ecuación de Stotzky (1965). Por su parte, las plántulas y los mixomicetes se registraron por triplicado, en el campo, a partir de parcelas de 1x1 m. En ambos casos, se calculó el Índice de Diversidad de Simpson en su forma intuitiva 1-D.

Las variables determinadas para toda la finca fueron analizadas de forma independiente para observar diferencias según uso de la tierra y dentro del parche boscoso, según zona. En estas ocasiones, una serie de análisis de varianza fueron llevados a cabo y en los casos positivos, pruebas post hoc de Tukey fueron usadas para determinar la direccionalidad de las diferencias, con un valor alfa de 0.05. Para el caso de pares categóricos, pruebas de chi cuadrado fueron usadas para evaluar diferencias con el mismo nivel de significancia anterior. Con las variables químicas de macro y micronutrientes, así como $\mathrm{pH}$, materia orgánica, saturación de acidez, densidad aparente y capacidad de campo, se realizó un análisis discriminante lineal. Con este análisis se buscó evaluar 
una reducción dimensional en la matriz original para determinar diferencias entre las secciones de la finca.

Resultados

En general, para FEIMA, los valores de textura del suelo determinados fueron de $59.9 \pm 13.5 \%$ de arena, $34.3 \pm 13.1 \%$ de limo y $5.7 \pm 2.6 \%$ de arcilla, lo cual permitió clasificar el suelo de esta zona como franco arenoso. Se observó que, de las tres secciones, el bosque tuvo el promedio más alto de arena (cuadro 1) y la sección de ganadería el más alto de limo. Algunas muestras en esta última sección se clasificaron como franco limosas. Dentro del bosque, la zona noroeste tuvo el promedio más alto de arena, incluso con zonas de suelo areno franco (figura 1). Ninguna de estas comparaciones en la textura según secciones de uso de la tierra o zonas dentro del bosque mostró significancia estadística.

La capacidad de campo promedio fue de 20.8土6.0 \% y fue mayor en la sección de ganadería

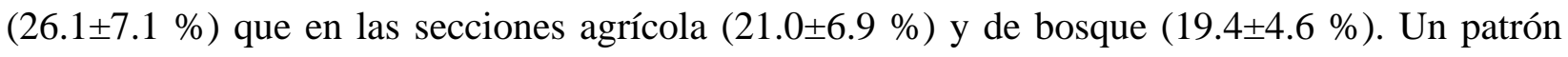
similar se encontró para el punto de marchitez permanente que fue mayor en la sección de ganadería $(11.4 \pm 2.6 \%)$ que en las secciones agrícola $(9.6 \pm 2.8 \%)$ y de bosque $(8.9 \pm 2.0 \%)$, con un promedio de $9.5 \pm 2.4 \%$. No se observaron diferencias significativas en estos valores según sección de uso de la tierra.

Cuadro 1. Valores porcentuales medios (y desviación estándar) determinados para las tres categorías de tamaño de partícula de suelo según sección de uso de la tierra y zona dentro del bosque en FEIMA. En negrita se muestran los valores más altos de cada tamaño de partícula por categoría espacial. 


\begin{tabular}{|c|c|c|c|}
\hline \multirow{2}{*}{$\begin{array}{l}\text { Sección } \\
\text { Zona del bosque }\end{array}$} & \multicolumn{3}{|c|}{ Textura, \% } \\
\hline & Arena & Limo & Arcilla \\
\hline Agrícola & 53.5 (15.6) & $35.0(15.7)$ & $5.5(1.9)$ \\
\hline Ganadería & $52.0(15.7)$ & $41.5(15.0)$ & $6.4(2.5)$ \\
\hline Bosque & $\mathbf{6 2 . 0}(11.7)$ & $32.3(11.4)$ & $5.7(2.9)$ \\
\hline Medio & $61.1(11.8)$ & $33.6(11.3)$ & $5.2(1.2)$ \\
\hline Sureste & $62.7(11.8)$ & $30.4(10.8)$ & $6.9(4.2)$ \\
\hline Noroeste & $64.2(13.6)$ & $31.2(13.8)$ & $4.5(0.2)$ \\
\hline
\end{tabular}

Los suelos también presentaron coloraciones claras de acuerdo con su matriz. En general, se encontraron entre 7.5YR y 10YR en el sistema de notación Munsell. El parámetro de valor de todas las muestras se encontró en el rango de los colores más oscuros (0-5) y según el chroma, los suelos mostraron expresión débil. Los horizontes superficiales observados fueron de los tipos Chernozem (36\%), Hórtico (53\%) y Fúlvico (9\%).

Se notaron tres sectores en la distribución de color del suelo en FEIMA. El primero coincidió con la sección agrícola y presentó en su totalidad suelos de color marrón oscuro, con una notación Munsell 7.5YR 3/4. El segundo sector estuvo representado por la sección de ganadería y partes de bosque en donde el suelo presentó el color café oscuro con notación Munsell 10YR 3/3. Finalmente, el tercer sector, con suelos color negro parduzco con notación Munsell 7.5YR 3/2, se observó solamente en partes del bosque noroeste y medio. No se observaron diferencias en el color según zona del bosque.

Con respecto a las propiedades químicas (ver cuadro 2), se pudo determinar que, de forma general, los suelos de la sección de bosque tuvieron valores más altos para la mayoría de los parámetros estudiados. Sin embargo, únicamente en el caso del manganeso se encontraron diferencias significativas según el uso actual de la tierra en FEIMA $(F(2,42)=4.48, P=0.017)$, con valores mucho más altos asociados con el suelo de uso agrícola. No fueron observadas diferencias en los parámetros químicos según zona del bosque. 


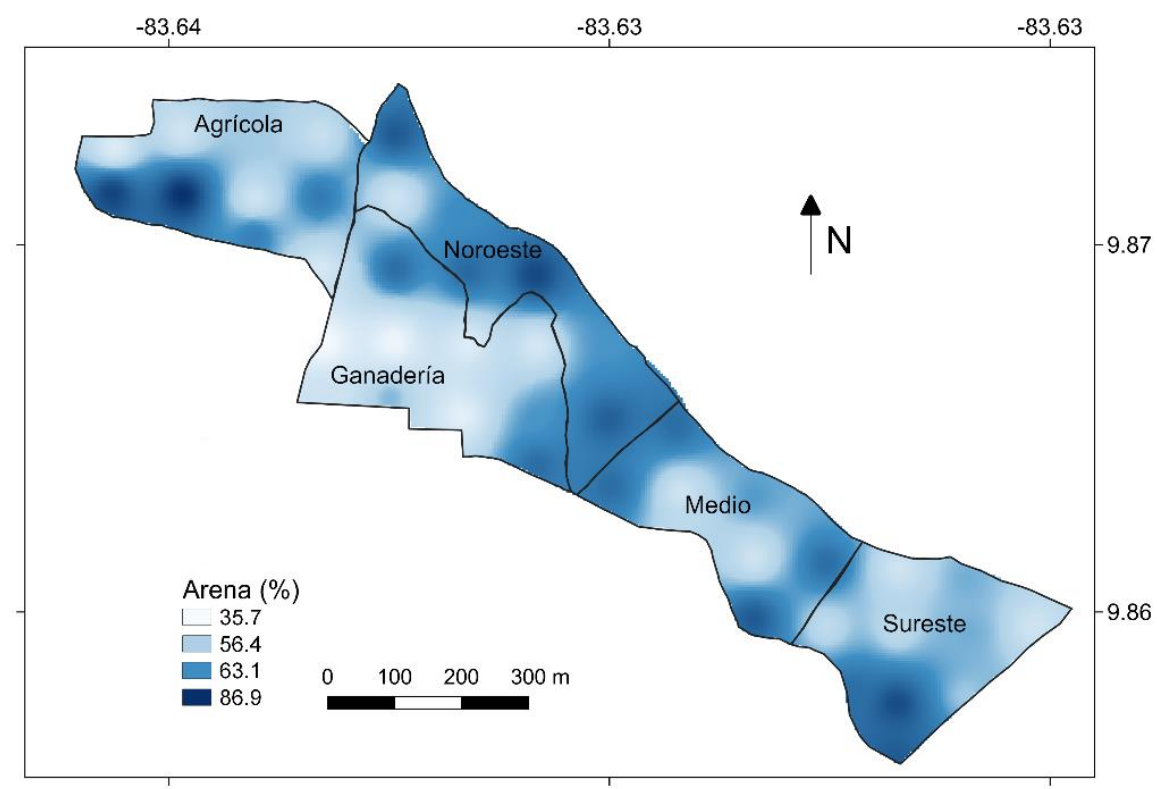

Figura 1. Mapa de FEIMA mostrando el porcentaje de arena determinado en las secciones agrícola y de ganadería y las tres zonas dentro de la sección de bosque.

En términos de acidez, únicamente un 9\% de las muestras analizadas se encontró en un rango problemático de $\mathrm{pH}$ y un $2 \%$ de las mismas mostró valores no adecuados de saturación de acidez. Los niveles de calcio y magnesio registraron buenos valores para todas las muestras y el potasio se encontró en niveles críticos en un 31\% de los casos, principalmente en la zona de bosque. El fósforo, el zinc y el manganeso por su parte, mostraron valores críticamente bajos en el 71\%, $51 \%$ y $5 \%$ de las muestras, respectivamente, y con mayor frecuencia en las secciones agrícola y de ganadería. A pesar de las anteriores diferencias, no se encontraron desigualdades en la fertilidad de la finca por sección espacial $\left(\square^{2}=1.22, d . f .=2, P=0.54\right)$.

El promedio de densidad aparente del suelo fue de $0.98 \pm 0.18 \mathrm{~g} / \mathrm{cm}^{3}$ en un rango de 0.6-1.4 $\mathrm{g} / \mathrm{cm}^{3}$ y se determinó que, para todas las muestras, este parámetro se encontró en el rango ideal para la respectiva clase textural. La mayor densidad aparente se registró en la sección agrícola $(1.07 \pm 0.12)$ y la menor en la zona de bosque $(0.94 \pm 0.17)$ con valores intermedios en la sección de ganadería. Dentro del bosque, la zona media mostró los valores más altos (1.02 \pm 0.16$)$, seguida de la zona sureste $(0.90 \pm 0.20)$ y noroeste $(0.88 \pm 0.16)$. De forma similar, la porosidad se observó en 
un rango entre 48.1-77.6\% con un promedio de 62.77 $\pm 6.86 \%$. Con un patrón inverso, los valores más bajos de porosidad se observaron en la sección agrícola $(59.27 \pm 4.74)$ y los más altos en la sección de bosque (64.23 77.28). Ninguna relación estudiada por sección o zona fue significativa.

Cuadro 2. Valores porcentuales medios (y desviación estándar) determinados para diferentes propiedades químicas del suelo según sección de uso de la tierra en FEIMA. En negrita se muestran los valores más altos para cada parámetro químico.

\begin{tabular}{lccc}
\hline Parámetro & Agrícola & $\begin{array}{c}\text { Sección } \\
\text { Ganadería }\end{array}$ & Bosque \\
\hline $\mathrm{pH}$ & $5.76(0.38)$ & $\mathbf{6 . 3 2}(0.23)$ & $6.03(0.40)$ \\
$\mathrm{CICE}$ & $18.13(2.81)$ & $\mathbf{2 1 . 1 4}(2.04)$ & $19.33(2.75)$ \\
$\mathrm{SA}, \%$ & $\mathbf{2 . 0 4}(3.09)$ & $0.62(0.31)$ & $0.90(0.99)$ \\
$\mathrm{MO}, \%$ & $2.78(1.30)$ & $2.88(1.21)$ & $\mathbf{3 . 2 6}(1.88)$ \\
$\mathrm{C}, \%$ & $1.51(0.50)$ & $1.62(0.69)$ & $\mathbf{1 . 9 4}(1.12)$ \\
$\mathrm{N}, \%$ & $0.16(0.04)$ & $0.17(0.07)$ & $\mathbf{0 . 2 1}(0.11)$ \\
$\mathrm{C} / \mathrm{N}$ & $\mathbf{9 . 2 7}(0.70)$ & $9.06(0.65)$ & $8.83(0.91)$ \\
$\mathrm{Ca}, \mathrm{cmol} / \mathrm{L}$ & $11.97(1.56)$ & $12.93(1.01)$ & $\mathbf{1 2 . 9 7}(2.11)$ \\
$\mathrm{Mg}, \mathrm{cmol} / \mathrm{L}$ & $5.53(2.19)$ & $\mathbf{7 . 5 2}(1.69)$ & $5.78(1.80)$ \\
$\mathrm{K}, \mathrm{cmol} / \mathrm{L}$ & $0.30(0.22)$ & $\mathbf{0 . 5 6}(0.54)$ & $0.42(0.29)$ \\
$\mathrm{P}, \mathrm{mg} / \mathrm{L}$ & $6.00(3.88)$ & $9.42(4.82)$ & $\mathbf{1 0 . 1 7}(6.00)$ \\
$\mathrm{Zn}, \mathrm{mg} / \mathrm{L}$ & $2.66(0.55)$ & $2.74(0.87)$ & $\mathbf{3 . 6 6}(1.70)$ \\
$\mathrm{Cu}, \mathrm{mg} / \mathrm{L}$ & $15.30(4.05)$ & $\mathbf{1 9 . 0 0}(3.60)$ & $15.01(5.09)$ \\
$\mathrm{Fe}, \mathrm{mg} / \mathrm{L}$ & $151.20(43.35)$ & $161.71(87.75)$ & $\mathbf{1 8 4 . 8 5}(70.01)$ \\
$\mathrm{Mn} * \mathrm{mg} / \mathrm{L}$ & $\mathbf{6 4 . 9}(39.13)$ & $36.42(22.82)$ & $36.89(20.95)$ \\
\hline
\end{tabular}

* diferencia significativa, $\mathrm{p}<0.05$

La velocidad de infiltración del agua en el suelo se encontró en un rango de 552-3405 mm/día, con un promedio de 1679 \$954 mm/día. En general, el suelo de la sección agrícola mostró los valores de velocidad más altos $(2361 \pm 225 \mathrm{~mm} /$ día) y el de ganadería los más bajos (733 257 $\mathrm{mm} /$ día). De forma similar, la infiltración acumulada se registró entre 12.5-84.1 cm de profundidad, 
con un promedio de $48.9 \pm 22.5 \mathrm{~cm}$. En este caso, los valores más altos se encontraron en la sección agrícola $(74.7 \pm 4.1 \mathrm{~cm})$ seguidos del bosque $(42.5 \pm 27.8 \mathrm{~cm})$ y la sección de ganadería $(18.0 \pm 7.7$ $\mathrm{cm})$. No se documentaron diferencias significativas en la velocidad de infiltración o la infiltración acumulada por zona del bosque.

El análisis discriminante lineal logró mostrar una separación de las tres secciones de la finca según las características de sus suelos (figura 2). Según este análisis, el 85\% de las muestras analizadas pudieron ser asociadas de forma predictiva con su respectiva sección de origen. Es relevante observar que el suelo del bosque se diferenció de los otros dos en relación con el eje primario, relacionado con micronutrientes y materia orgánica. Sin embargo, es interesante observar que la diferenciación observada entre los suelos agrícolas y de la sección de ganadería se explicó de forma secundaria, por textura y acidez. En el cuadro 3 se observa de forma resumida la direccionalidad en las características de suelo según sección estudiada.

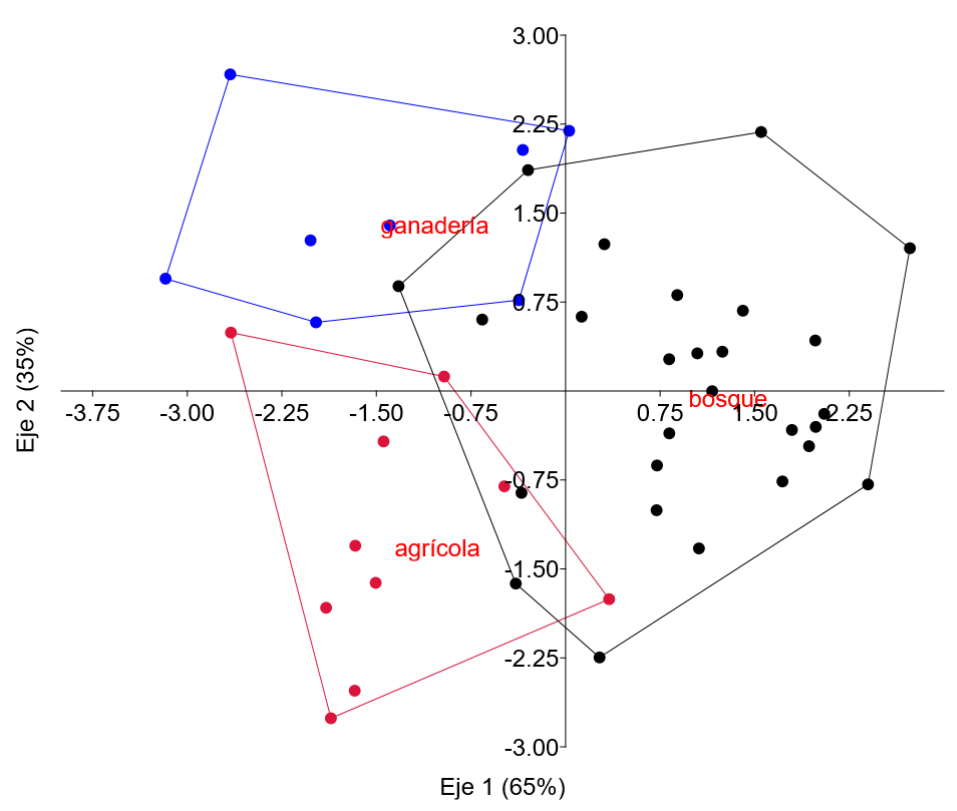

Figura 2. Biplot del análisis discriminante lineal mostrando la separación de las tres secciones de FEIMA según el análisis multivariado realizado con una serie de variables independientes

La respiración de suelo se determinó en un rango de 35.0-61.7 $\mu \mathrm{g}$ C-CO2/gh, con un promedio de $46.3 \pm 10.0 \mu \mathrm{g}$ C-CO2/gh. Con esta variable, la sección sureste del bosque presentó 
valores más bajos (37.2 $\pm 2.7 \mu \mathrm{g}$ C-CO2/gh) que la sección media $(52.3 \pm 9.0 \mu \mathrm{g} \mathrm{C}-\mathrm{CO} 2 / \mathrm{gh})$ o la noroeste $(49.5 \pm 10.0 \mu \mathrm{g} \mathrm{C}-\mathrm{CO} 2 / \mathrm{gh})$, mostrando diferencias significativas $(F(2,15)=6.03, P=0.01)$. La diversidad de plántulas según el Índice de Simpson se estimó en $0.51 \pm 0.21$ con un rango entre 0.37-0.66 y a pesar de que hubo variación entre las zonas media $(0.66 \pm 0.24)$, sureste $(0.46 \pm 0.25)$ y noroeste $(0.45 \pm 0.11)$, no se observaron diferencias significativas. La misma ausencia de diferencias fue observada para la diversidad de mixomicetes, que fue calculada con un promedio de $0.75 \pm 0.06$ en un rango entre $0.61-0.92$. En este último caso, los valores tuvieron muy poca variación entre la zona noroeste $(0.76 \pm 0.10)$, media $(0.75 \pm 0.02)$ y sureste $(0.74 \pm 0.02)$. No se observaron relaciones significativas entre las tres variables de respuesta y los parámetros de suelo determinados para el parche boscoso.

Cuadro 3. Resumen de la direccionalidad en algunos de los parámetros estudiados según sección de uso de la tierra en FEIMA.

\begin{tabular}{lccc}
\hline Parámetro & Agrícola & $\begin{array}{c}\text { Sección } \\
\text { Ganadería }\end{array}$ & Bosque \\
\hline Textura & intermedia & más limosa & más arenosa \\
Color & menos oscuro & intermedio & más oscuro \\
Capacidad de & intermedia & más alta & más baja \\
campo & & & \\
Propiedades & macro/micronutrientes & macro/micronutrientes en & macro/micronutrientes \\
químicas & en rango bajo, materia & rango medio-alto, materia & en rango alto, materia \\
& orgánica baja, pH bajo & orgánica intermedia, pH & orgánica alta, pH \\
& & alto & intermedio \\
Densidad aparente & más alta & intermedia & más baja \\
Porosidad & más baja & intermedia & más alta \\
Velocidad de & más alta & más baja & intermedia \\
infiltración & & & \\
\hline
\end{tabular}




\section{Discusión}

En términos generales, los resultados de este estudio mostraron que después de un periodo de 30 años, el suelo de FEIMA ha cambiado de forma diferenciada según el régimen de uso de la tierra al que ha estado sujeto. Si bien esta diferenciación se observó en mayor grado para algunas de las variables analizadas (p.e. nivel de manganeso), es fundamental establecer la linealidad de las variaciones en el tiempo con el fin de ofrecer insumos importantes para la gestión adecuada del sitio de estudio (ver Bautista-Cruz et al., 2005). Por ello ha sido interesante observar que el bosque, fue en muchos de los casos, el sistema estudiado donde se encontraron valores extremos; y que la zona originalmente destinada para sistemas silvopastoriles mostró valores intermedios. Esos resultados sugieren que la cobertura forestal ha tenido un efecto amortiguador sobre los procesos de transformación del suelo en la finca estudiada.

Es importante recordar que estos procesos de cambio continúan todavía, por lo que la velocidad de modificación de estas variables es también relevante para un análisis integrado (Hall, 2012). De forma similar, la variación espacial en la magnitud asociada con las variables de estudio, más allá de su relación con el uso de la tierra, es importante para analizar homogeneidad dentro de sistemas presumiblemente estables (ver Sparling, Schipper, Bettjeman, y Hill, 2004). De esta forma, las variaciones observadas en la textura, color y propiedades hidráulicas del suelo a lo largo del sitio de estudio claramente han reflejado el manejo diferente en cada sección de FEIMA, pero también han mostrado diferenciaciones dentro de cada unidad de uso del suelo. Estas pequeñas oscilaciones en la magnitud de las variables tienen el potencial de servir como modelos de evaluación de los procesos asociados con la formación de microzonas (Ettema \& Wardle, 2002).

Es interesante observar que, en términos de color, el suelo de FEIMA se encontró entre los valores 7.5YR y 10YR en el sistema de notación Munsell. Estos colores son comunes cuando los suelos se encuentran en estados iniciales a intermedios de alteración bajo ambientes de oxidación y se relacionan con niveles bajos o medios de materia orgánica (Fernandez, Schulze, Coffin, y Van Scoyoc, 1988), algo observado en este estudio (entre 2.2-3.7\%). También es notable que, según los valores químicos obtenidos, el $73 \%$, el $71 \%$ y el $53 \%$ de las muestras estudiadas mostraron desbalances en las relaciones $\mathrm{Ca} / \mathrm{K}, \mathrm{Ca}+\mathrm{Mg} / \mathrm{K}$ y $\mathrm{Mg} / \mathrm{K}$, respectivamente. Lo anterior pareciera ser producto de una tendencia generalizada de bajos valores de potasio en el suelo. Sin embargo, 
tomando en cuenta el origen del suelo analizado y la capacidad del potasio de lixiviarse (Alfaro, Jarvis, y Gregory, 2004), tal resultado no es inesperado.

De forma similar, los valores sistemáticamente bajos de fósforo y zinc, a pesar de corresponder con valores similares obtenidos en estudios previos en el Neotrópico para suelos franco-arenosos (ver Alvarado \& Raigosa, 2012), parecen denotar además el origen pobre del suelo inicial. Es interesante observar, que de forma esperada, el valor ligeramente más alto está asociado con la sección de bosque donde la dinámica micorrícica debería de ser más activa (Janos, 1980). De igual forma, a pesar de que es esperable que la densidad aparente sea menor (y la porosidad mayor) en el bosque, por un menor efecto de compactación antropogénica y mayor actividad biológica (ver Lal, 1988), es curioso no haber observado diferencias significativas entre secciones de uso de la tierra. Lo anterior simplemente sugiere que el periodo de tiempo bajo el cual la finca ha estado sujeta a manejo diferenciado no ha sido suficiente para generar estas variaciones esperadas.

Un fenómeno similar pareciera haber sucedido con los parámetros de infiltración determinados, que, a pesar de no mostrar todavía diferencias entre secciones de uso de la tierra, han empezado a divergir entre ellos. En este caso, sin embargo, pareciera que el efecto de compactación por la presencia animal en la zona de ganadería ha modificado visiblemente las propiedades de infiltración del suelo. Este efecto anterior ha sido ampliamente documentado con anterioridad (p.e. Bezkorowajnyj, Gordon, \& McBride, 1993) y era esperable. Es relevante, sin embargo, que a pesar de que el suelo del bosque mostró valores intermedios para esta variable, otros autores han comunicado valores más altos para estos sistemas que para zonas agrícolas adyacentes (ver Mathur, Singh, \& Gupta, 1982; Ellison et al., 2017). En el caso del presente estudio, un efecto externo que pudo haber influenciado tales mediciones fue una reducción de alrededor de un $80 \%$ en la precipitación natural (datos propios no publicados) para los meses de estudio durante el año en que se realizaron las pruebas. Esta precipitación disminuida seguramente afectó en mayor grado una sección sin cobertura forestal como la agrícola, y facilitó valores más altos para las variables estudiadas.

En todo caso y de forma paralela a la anterior observación, los datos registrados en este estudio han sugerido dos patrones en la finca estudiada. El primero es que los suelos de la sección de bosque han sido más resilientes a los diferentes agentes de cambios en el tiempo que los suelos 
en las otras dos secciones de la finca. La poca documentación de diferencias en las variables para las tres zonas estudiadas dentro del bosque, también mostraron que desde el punto de vista funcional, todo el bosque es una unidad homogénea. El segundo patrón observado es que, después de 30 años, el uso destinado de la tierra tuvo mayor potencial para determinar diferencias entre las variables de estudio, que el posicionamiento geográfico y potencial heterogeneidad inicial de depósito de materiales. Lo anterior, por cuanto las diferencias espaciales en las características del suelo son pocas cuando se analizan con un modelo de nulidad espacial, pero empiezan a ser evidentes al ser estudiadas según las diferentes secciones de uso de la tierra.

Agradecimientos

Se agradece la colaboración de Lolita Durán, Alex Murillo y Werner Rodríguez por permitir la realización de este proyecto y el de María Araya por apoyo técnico. El financiamiento ha sido otorgado por la Vicerrectoría de Investigación de la Universidad de Costa Rica (731-B8-034). Se reconoce y agradece el apoyo de Aldo Quesada Chacón durante la toma de datos en el campo y la colaboración logística ofrecida por la Sede del Atlántico de la Universidad de Costa Rica.

\section{Referencias}

Alfaro, M. A., Jarvis, S. C., \& Gregory, P. J. (2004). Factors affecting potassium leaching in different soils. Soil Use and Management, 20, 182-189. https://doi.org/10.1079/SUM2004249

Alvarado, A., \& Raigosa, J. (2012). Nutrición y fertilización forestal en regiones tropicales. San José, Costa Rica: Asociación Costarricense de la Ciencia del Suelo.

Barnes, A. D., Allen, K., Kreft, H., Corre, M. D., Jochum, M., Veldkamp, E., ... \& Brose, U. (2017). Direct and cascading impacts of tropical land-use change on multi-trophic biodiversity. Nature Ecology \& Evolution, 1, 1511-1519. https://doi.org/10.1038/s41559-017-0275-7

Bautista-Cruz, A., Gutiérrez-Castorena, M. D. C., Castillo-Sánchez, R. D., \& Etchevers-Barra, J. D. (2005) Cronosecuencia de un suelo y su clasificación en un área originalmente ocupada por 
bosque mesófilo de montaña. Terra Latinoamericana, 23, 147-157. Recuperado de: http://www.redalyc.org/articulo.oa?id=57323201

Bezkorowajnyj, P. G., Gordon, A. M., \& McBride, R. A. (1993). The effect of cattle foot traffic on soil compaction in a silvo-pastoral system. Agroforestry Systems, 21, 1-10. https://doi.org/10.1007/BF00704922

Cavender, J. C., \& Raper, K. B. (1965). The Acrasieae in nature. I. Isolation. American Journal of Botany, 52, 294-296. https://doi.org/10.2307/2439943.

Codur, A. M., \& Watson, J. 2018. Climate smart or regenerative agriculture? Defining climate policies based on soil health. GDAE Climate Policy Brief, 9, 1-10. Recuperado de: http://ase.tufts.edu/gdae/Pubs/climate/ClimatePolicyBrief9.pdf

Consejo Universitario. (2009, 2 de junio). Acta de la sesión No. 5357. Disponible en: http://www.cu.ucr.ac.cr/uploads/tx_ucruniversitycouncildatabases/minute/2009/5357.pdf

Ellison, D., Morris, C. E., Locatelli, B., Sheil, D., Cohen, J., Murdiyarso, D., ... \& Sullivan, C. (2017). Trees, forests and water: Cool insights for a hot world. Global Environmental Change, 43, 51-61. https://doi.org/10.1016/j.gloenvcha.2017.01.002

Ettema, C. H., \& Wardle D. A. (2002). Spatial soil ecology. Trends in Ecology \& Evolution, 17, 177-183. https://doi.org/10.1016/S0169-5347(02)02496-5

Fernandez, R. N., Schulze, D. G., Coffin, D. L., \& Van Scoyoc, G. E. (1988). Color, Organic Matter, and Pesticide Adsorption Relationships in a Soil Landscape. Soil Science Society of America Journal, 52, 1023-1026. https://doi.org/10.2136/sssaj1988.03615995005200040023x

Forsythe, W. (1985). Física de Suelos: Manual de Laboratorio. San José, Costa Rica: IICA.

Wall, D. H., Ritz, K., Six, J., Strong, D. R., \& van der Putten, W. H. (Eds.) (2012). Soil ecology and ecosystem services. Oxford: Oxford University Press.

Janos, D. P. (1980). Mycorrhizae influence tropical succession. Biotropica, 12, 56-94. https://doi.org/10.2307/2388157 
Kessler, J., \& Oosterbaan, R. J. (1974). Determining Hydraulic Conductivity of Soils. Drainages Principles and Applications. Wagingen, Holanda: International Institute for Land Reclamation and Improvement.

Lal, R. (1988). Effects of macrofauna on soil properties in tropical ecosystems. Agriculture, Ecosystems \& Environment, 24, 101-116. https://doi.org/10.1016/0167-8809(88)90059-X

Lal, R. (2016). Soil health and carbon management. Food and Energy Security, 5, 212-222. https://doi.org/10.1002/fes3.96

Mathur, R. N., Singh, R. P., \& Gupta, M. K. (1982). Comparative Study of Infiltration in Soils under Forest Cover and Agriculture in Temperate Climate. Indian Forester, 108, 648-652. Recuperado de: http://www.indianforester.co.in/index.php/indianforester/article/view/10971

Méndez, J. C., Bertsch, F. (2012). Guía para la interpretación de la fertilidad de los suelos de Costa Rica. San Pedro de Montes de Oca, Costa Rica: Asociación Costarricense de Ciencia del Suelo.

Moebius-Clune, B., Moebius-Clune, D., Gugino, B. K., Idowu, O. J., Schindelbeck, R. R., Ristow, A. J., ... \& Abawi, G. S. (2016). Comprehensive Assessment of Soil Health. Geneva, NY: Cornell University.

Resolución R-27-2014. La Gaceta Universitaria, San Pedro de Montes de Oca, Costa Rica, 10 de febrero de $2014 . \quad$ Recuperado de: http://www.cu.ucr.ac.cr/uploads/tx_ucruniversitycouncildatabases/officialgazette/2014/a032014.pdf\#page $=1$

Salmon, S. (2018). Changes in humus forms, soil invertebrate communities and soil functioning with forest dynamics. Applied Soil Ecology, 123, 345-354. https://doi.org/10.1016/j.apsoil.2017.04.010

Saxton, K. E., Rawls, W., Romberger, J. S., \& Papendick, R. I. (1986). Estimating generalized soilwater characteristics from texture. Soil Science Society of America Journal, 50, 1031-1036. https://doi.org/10.2136/sssaj1986.03615995005000040039x 
Sibaja-Matarrita, R., Barboza-Chinchilla, L., \& Rojas, C. (2018). ¿Pueden los micetozoos ser usados como indicadores de salud del suelo en el contexto agrícola de Costa Rica? Revista de Ciencias Ambientales, 52, 161-174. https://doi.org/10.15359/rca.52-1.9

Sparling, G. P., Schipper, L. A., Bettjeman, W, \& Hill, R. (2004). Soil quality monitoring in New Zealand: practical lessons from a 6-year trial. Agriculture, Ecosystems \& Environment, 104, 523 534. https://doi.org/10.1016/j.agee.2004.01.021

Stephenson, S. L., Fiore-Donno, A. M., \& Schnittler, M. (2011). Myxomycetes in soil. Soil Biology and Biochemistry, 43, 2237-2242. https://doi.org/10.1016/j.soilbio.2011.07.007

Stocking, M. A. (1988). Assessing Vegetative Cover and Management Effects. En: R. Lal (Ed.), Soil Erosion Research Methods (pp.211-234). Ankeny, Iowa: Water Conservation Society.

Stotzky, G. (1965). Microbial respiration. In: C. A. Black, D. D. Evans, J. L. E. Ensminger, \& F. E. Clark, (Eds.). Methods of soil analysis. Part 2. Chemical and microbial properties (pp. 15501572). Madison, Wisconsin: American Society of Agronomy.

Wieder, W. R., Allison, S. D., Davidson, E. A., Georgiou, K., Hararuk, O., He, Y., Hopkins, F., ... \& Xu, X. (2015). Explicitly representing soil microbial processes in Earth system models, Global Biogeochemical Cycles, 29, 1782-1800. https://doi.org/10.1002/2015GB005188

Whiting, D., Wilson, C., \& Card, A. (2003). Estimating Soil Texture: Sandy, Loamy, or Clayey? Colorado Master Gardener Fact Sheets, S14, 1-5. 